\title{
Ações de educação em saúde para agentes comunitários de saúde
}

\author{
Jéssica Samara Oliveira TOLOMEU ${ }^{1(*)}$ \\ Paola Aparecida Alves FERREIRA ${ }^{2 *}$ \\ Angélica Pataro REIS ${ }^{3}$ \\ Daisy de Rezende Figueiredo FERNANDES ${ }^{4}$ \\ Leida Calegário de OLIVEIRA ${ }^{5}$ \\ ${ }^{1}$ Enfermeira,Pesquisadora,Universidade Federal dos Vales do Jequitinhonha e Mucuri-UFVJM. \\ jessica.samarat@hotmail.com \\ ${ }^{2}$ Nutricionista, Pesquisadora, UFVJM. paola.dtna@gmail.com \\ ${ }^{3}$ Bioquímica, Doutora. Docente Depto Ciências Básicas -FCBS /UFVJM. angelicapataro@hotmail.com \\ ${ }^{4}$ Enfermeira, Doutoranda. Docente Depto Enfermagem -FCBS/UFVJM. daisygouveia@oi.com.br \\ ${ }^{5}$ Bióloga, Doutora. Docente do Departamento de Farmácia /FCBS/UFVJM. leida@ufvjm.edu.br
}

(*) Estes autores contribuíram igualmente para a realização deste trabalho.

Recebido em: 30/05/2013 - Aprovado em: 12/07/2013 - Disponibilizado em: 15/08/2013

\section{Resumo:}

Os agentes comunitários de saúde constituem estratégia de aprimoramento e consolidação da ESF, atuando como elo facilitador que possibilita a confiança e o vínculo entre a equipe de saúde e a comunidade assistida. Baseado na importância do trabalho dos ACS na equipe de saúde da família, este trabalho teve como objetivo realizar um levantamento sobre as ações de educação continuada ou de educação permanente realizadas no país para capacitação destes profissionais, bem como do perfil destas ofertados aos ACS no município de Diamantina, MG. Foi realizada uma pesquisa nas bases de referências bibliográficas MEDLINE, LILACS e SciElo para o período de 2008 a 2012. Utilizando os descritores "Agentes comunitários de saúde", "ACS", "capacitação em serviço", "educação em saúde" e "treinamento em serviço" foram levantados 19 artigos científicos completos e originais. A análise dos dados obtidos nos artigos, bem como nas ESFs do município de Diamantina, MG, nos mostrou que em ambas as situações houve o predomínio de ações de educação ofertadas na modalidade presencial. Na análise dos artigos observou-se que as capacitações realizadas no período tratavam de temas bastante diversificados e ocorreram em pequeno número, porém em vários estados brasileiros. Em Diamantina, MG, o número de capacitações também foi muito pequeno, tendo sido realizadas em horário de trabalho dos ACS. É necessário que se invista em ações de capacitação para os ACS, de forma sistematizada,uma vez que são eles quem mantêm o contato estreito com os usuários do sistema.

Palavras - chave: Agentes Comunitários de Saúde. Educação em Saúde. Capacitação em Serviço.

\section{Health education actions for community health agents}

\begin{abstract}
:
Community health agents constitute a strategy for the improvement and consolidation of the Family Health Strategy (FHS), acting as a facilitating link that enables the build-up of confidence and bond between the health team and the assisted community. Based on the importance of the work of Community Health Agents (CHA) in the family health team, the objective of this paper was to conduct a survey about the actions in continuing education or permanent education undertaken in the country for the qualification of these professionals, as well as the profile of such actions provided to the CHA in the municipality of Diamantina, Minas Gerais state, Brazil. Research was carried out in the bibliographic reference databases MEDLINE, LILACS, and SciElo for the period from 2008 to 2012. By using the descriptors "Community Health Agents", "CHA", "in-service qualification", "health education", and "inservice training", 19 complete and original scientific articles were reviewed. The analysis of the data obtained from the articles, as well as from the Family Health Strategies in the municipality of Diamantina, Minas Gerais state, showed us that in both situations there was a predominance of education actions offered face-to-face. The analysis of the articles also showed that the qualification activities carried out in the above-mentioned period covered a wide range of themes, and were few in number. However, they occurred in several Brazilian states. In Diamantina, Minas Gerais state, the number of
\end{abstract}


qualification activities was also very small, and took place during the working hours of the CHA. It is necessary to invest, in a systematic manner, in qualification actions for the Community Health Agents, since they are the ones who maintain a close contact with the users of the health system.

Key Words: Community Health Agents. Health Education. In-Service Qualification.

\section{Introdução}

\section{A Atenção Básica caracteriza-se por um} conjunto de ações de saúde, no âmbito individual e coletivo, que abrangem a promoção e a proteção da saúde, a prevenção de agravos, o diagnóstico, o tratamento, a reabilitação e a manutenção da saúde (DIAS et al., 2011). É desenvolvida por meio do exercício de práticas gerenciais e sanitárias democráticas e participativas, sob forma de trabalho em equipe, dirigidas a populações de territórios bem delimitados, pelas quais assume a responsabilidade sanitária, considerando a dinamicidade existente no território em que vivem essas populações (MINISTÉRIO DA SAÚDE, 2006).

A estratégia Saúde da Família (ESF) reafirma e incorpora os princípios básicos do Sistema Único de Saúde (SUS), isto é, a universalização, descentralização, integralidade das ações e participação da comunidade. Está estruturada a partir da Unidade de Saúde da Família (USF), com equipe multiprofissional, que passa a ser responsável pelo acompanhamento permanente da saúde de um número determinado de indivíduos e famílias que moram no espaço territorial próximo, possibilitando o estabelecimento de vínculos de compromisso e de co-responsabilidade entre os profissionais de saúde e a população (MINISTÉRIO DA SAÚDE, 1999).

Estrutura-se incorporando as diretrizes de universalização, descentralização e participação da comunidade. Anunciase com princípios complementares: caráter substitutivo ao modelo tradicional de atenção primária, antes centrada prioritariamente na relação médico-paciente e no atendimento individual; integralidade e hierarquização; territorialização e cadastramento da clientela; equipe multiprofissional composta por um médico generalista ou médico de família, um enfermeiro, um auxiliar de enfermagem e de quatro a seis agentes comunitários de saúde (MINISTÉRIO DA SAÚDE, 2001).

Cada equipe de saúde da família, é responsável por no máximo 4.000 habitantes, sendo a média recomendada de 3.000 habitantes, com jornada de trabalho de 40 horas semanais para 
todos os seus integrantes. São atribuições comuns a todos os profissionais, garantir a integralidade da atenção por meio da realização de ações de promoção da saúde, prevenção de agravos e curativas e da garantia de atendimento da demanda espontânea, da realização das ações programáticas e de vigilância à saúde. (MINISTÉRIO DA SAÚDE, 2006). Os agentes comunitários de saúde (ACS) integram a Equipe de Saúde da Família, atuando como um braço da Estratégia de Saúde da Família na residência do usuário. $\mathrm{O}$ ACS desponta como o elo facilitador que possibilita a confiança e o vínculo entre a equipe de saúde da famíliae a comunidade assistida.

Silva e Dalmaso (2002) entendem que o saber sistematizado e os instrumentos existentes na área da saúde não são suficientes para suprir as necessidades de trabalho dos agentes, tanto no que se refere à abordagem familiar, como em relação ao contato com situações de vida precária. A falta de saberes sistematizados para o pólo mais político e de assistência social acaba fazendo com que os ACS trabalhem mais com o senso comum e com a religião. Ressaltam ainda a variedade de expectativas existentes sobre a função do ACS, que ora se vê como educador em saúde, ora como organizador de acesso, como "olheiro" da equipe na identificação de necessidades.

Sendo assim, baseado na importância do trabalho dos ACS na equipe de saúde da família, objetivou-se realizar um levantamento sobre as ações de educação continuada ou de educação permanente realizadas no país para capacitação destes profissionais, bem como do perfil dos cursos e outras modalidades de educação ofertados a estes profissionais no município de Diamantina, MG.

\section{Metodologia}

Para viabilizar esse estudo foi feita uma pesquisa nas bases de referências bibliográficas MEDLINE, LILACS e SciElo em maio de 2013, para os cinco últimos anos (2008 e 2012). Foram levantados todos os artigos científicos completos e originais publicados no período de 2008 a 2012 e que tratavam da educação em saúde para os ACS no Brasil. Neste sentido, excluíram-se deste levantamento todos os trabalhos de monografias, dissertações, teses e resumos publicados em anais de eventos, bem como os artigos fornecidos em duplicata ou triplicata pelas bases de referências bibliográficas. Os descritores utilizados para busca nas três bases de dados ("Agentes comunitários de saúde", "ACS”, "capacitação em serviço", 
"educação em saúde" e "treinamento em serviço") foram definidos a partir de consulta ao "Descritores em Ciências da Saúde" na Biblioteca Virtual em Saúde. Os artigos foram lidos e a partir daí selecionouse aqueles que integrariam este trabalho por tratar do tema aqui abordado. Chegou-se assim a um quantitativo de 19 artigos completos que se encontravam disponíveis nas bases de dados. $\mathrm{Na}$ sequência, construiu-se uma planilha que foi alimentada a partir do levantamento dos seguintes dados nos artigos: ano de publicação; modalidade da ação (presencial, à distância); tema; aprovação por CEP; local.

Após a realização desta revisão, fez-se um levantamento junto às Estratégias de Saúde da Família do município de Diamantina, MG, para avaliar o perfil das capacitações ofertadas pelas esferas municipal, estadual ou mesmo federal aos ACS.

Os dados foram obtidos através da análise de relatórios, listas de presença e livros de atas referentes aos cursos de capacitação. Foram levantados os seguintes dados: Ano de treinamento; modalidade do treinamento; tema; horário de realização da capacitação. O presente trabalho seguiu as diretrizes e normas regulamentadoras envolvendo seres humanos, de acordo com a
Resolução do Conselho Nacional de Saúde - CNS 196/96.

\section{Resultados}

Para a realização deste trabalho, convidou-se todas as ESFs do município de Diamantina/MG (n=9) para participar. Destas, apenas uma não aceitou participar. Sendo assim, os resultados apresentados referem-se aos dados obtidos de oito Estratégias.

A leitura dos artigos selecionados $(n=19)$ para o desenvolvimento deste estudo permitiu-nos observar que $89,4 \%$ das atividades de educação em saúde ofertadas aos ACS foram oferecidas na modalidade presencial, 5,3\% à distância, enquanto que $5,3 \%$ aconteceram de forma mista, possuindo atividades presenciais e à distância.

Em relação ao tipo de treinamento oferecido, observou-se que $36,8 \%$ foram cursos de capacitação, 26,3\% foram realizados na modalidade oficina, $10,5 \%$ foram encontros de atualização, 5,3\% consistiram em treinamentos, enquanto $21,1 \%$ das atividades de educação continuada foram realizadas em outras modalidades.

Em relação aos aspectos éticos para o desenvolvimento de pesquisa científica, observou-se relato dos autores mencionando aprovação por Comitê de Ética em Pesquisa em 52,6\% das publicações. Outro aspecto importante é que em apenas 10,5\% dos artigos há a 
menção de que as atividades de capacitação tenham sido realizadas durante o horário de trabalho dos ACS. Provavelmente, os autores esqueceram-se de relatar a existência de um parecer favorável, uma vez que a não aprovação deste consistiria em ato ilegal, infringindo a Resolução $\mathrm{n}^{\mathrm{o}}$ 196/96 do Conselho Nacional de Saúde que regulamenta a pesquisa envolvendo seres humanos.

Observou-se ainda que as atividades de capacitação vêm sendo realizadas em diversas regiões do país, tendo um predomínio de ações no Estado de São Paulo (Fig.1).

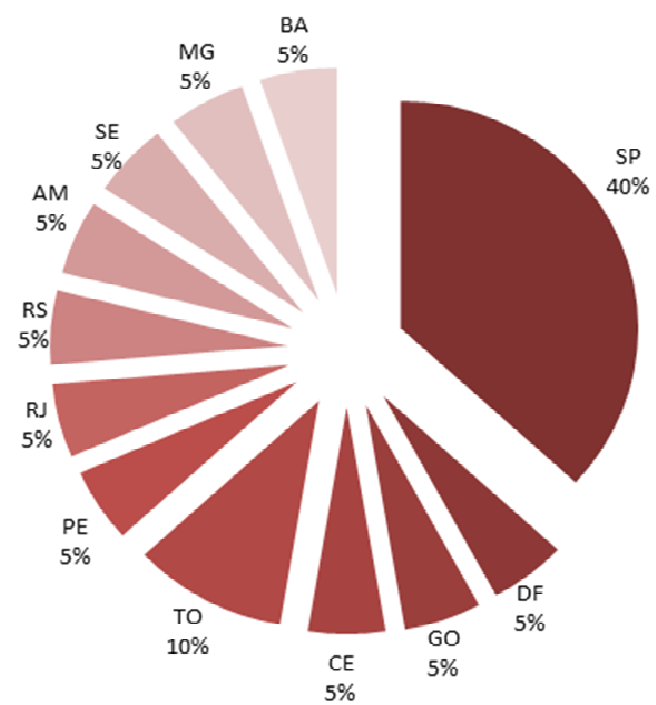

FIGURA 1 - Distribuição dos artigos publicados no período de 2008 a 2012 que tratam de educação em saúde para Agentes Comunitários de Saúde por região do país.

Sobre os assuntos abordados, foram observadas uma grande diversidade de temas que, segundo relatos dos autores, foram decididos de acordo com as necessidades e interesses do grupo a ser capacitado (Tab.1)

TABELA 1 - Temas tratados nas ações de educação em saúde ofertadas aos Agentes Comunitários de Saúde nos artigos referentes ao tema publicados no período de 2008 a 2012.

\begin{tabular}{|c|c|}
\hline ASSUNTOS ABORDADOS & $\%$ \\
\hline 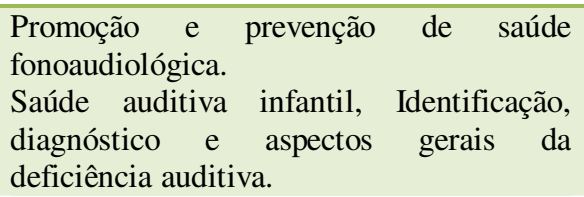 & 15,8 \\
\hline $\begin{array}{l}\text { Doenças prevalentes na infância; Doenças } \\
\text { respiratórias infantis, Asma em crianças, } \\
\text { imunização; avaliação de crescimento; } \\
\text { aleitamento materno; prevenção de } \\
\text { violência doméstica; prevenção do câncer } \\
\text { infantil. }\end{array}$ & 15,8 \\
\hline $\begin{array}{l}\text { Gestão em saúde e processo de trabalho } \\
\text { dos ACS; promoção da saúde e prevenção } \\
\text { de doenças; situações de risco ambiental e } \\
\text { sanitário, Biodiversidade; lixo; água; } \\
\text { consumo responsável; as zoonoses e } \\
\text { convivência saudável. }\end{array}$ & 10,5 \\
\hline $\begin{array}{l}\text { Integração do grupo; trabalho em equipe; } \\
\text { reconhecimento do território; educação e } \\
\text { saúde; atribuições do agente comunitário } \\
\text { de saúde; políticas de saúde no Brasil; } \\
\text { família; conceito de território; diagnóstico } \\
\text { cadastro; direitos; participação e } \\
\text { mobilização comunitária; ciclo vital. }\end{array}$ & 5,3 \\
\hline $\begin{array}{l}\text { Leitura relacional do Almanaque da } \\
\text { Dengue;memória social e epidemias de } \\
\text { dengue; culpabilização da vítima e } \\
\text { informação; o ACS: informante não } \\
\text { autorizado. }\end{array}$ & 5,3 \\
\hline $\begin{array}{l}\text { Compreensão do ciclo de vida do vetor e } \\
\text { do parasito de doenças como: } \\
\text { oncocercose, mansonelose, malária, } \\
\text { leishmaniose, esquistossomose e doença } \\
\text { de Chagas. }\end{array}$ & 5,3 \\
\hline $\begin{array}{l}\text { Cultura, território, política, cuidado , } \\
\text { informação, educação e planejamento em } \\
\text { saúde. }\end{array}$ & 5,3 \\
\hline $\begin{array}{l}\text { Noções básicas de utilização do hardware } \\
\text { e do software. }\end{array}$ & 5,3 \\
\hline Saúde mental e saúde da família. & 5,3 \\
\hline $\begin{array}{l}\text { Intervenção educacional sobre aleitamento } \\
\text { materno. }\end{array}$ & 5,3 \\
\hline $\begin{array}{l}\text { Saúde da criança e idoso; violência contra } \\
\text { a criança, mulher e idoso; atribuições dos } \\
\text { ACS; ética profissional; comunicação e } \\
\text { trabalho em equipe. }\end{array}$ & 5,3 \\
\hline
\end{tabular}


Prevenção do câncer cérvico-uterino.

Relações de gênero, diversas formas de 5,3 violência; direitos sexuais e reprodutivos; práticas cotidianas de trabalho dos ACS.

Conhecimentos sobre saúde bucal.

Buscando conhecer melhor a realidade dos

Agentes Comunitários de Saúde do município de Diamantina, MG, para nos nortear sobre ações a serem desenvolvidas para a capacitação destes sujeitos, decidiuse fazer um levantamento do número e perfil das ações de educação em saúde ofertadas no ano de 2012 pelo município aos mesmos. Para tanto, fez-se uma busca nos documentos (listas de presença, livros de atas, relatórios) das nove ESFs do município de Diamantina. A análise dos dados permitiu-nos observar que em 25,0\% das ESFs do município de Diamantina, os ACS foram contemplados com atividades de educação continuada no ano de 2012. Entretanto, como tal, são ações esporádicas, realizadas de acordo com a necessidade e com a disponibilidade de preceptores. Em $75,0 \%$ das ESFs não foi relatada a oferta de atividades de capacitação para os ACS no ano de 2012 (Fig.2).

Dentre as ações de educação continuada ofertadas no ano de 2012 aos ACS, o tema trabalhado em $50,0 \%$ destas foi "Alimentação complementar para crianças de até dois anos". Todas as atividades de capacitação foram realizadas em horário de trabalho e na modalidade presencial.

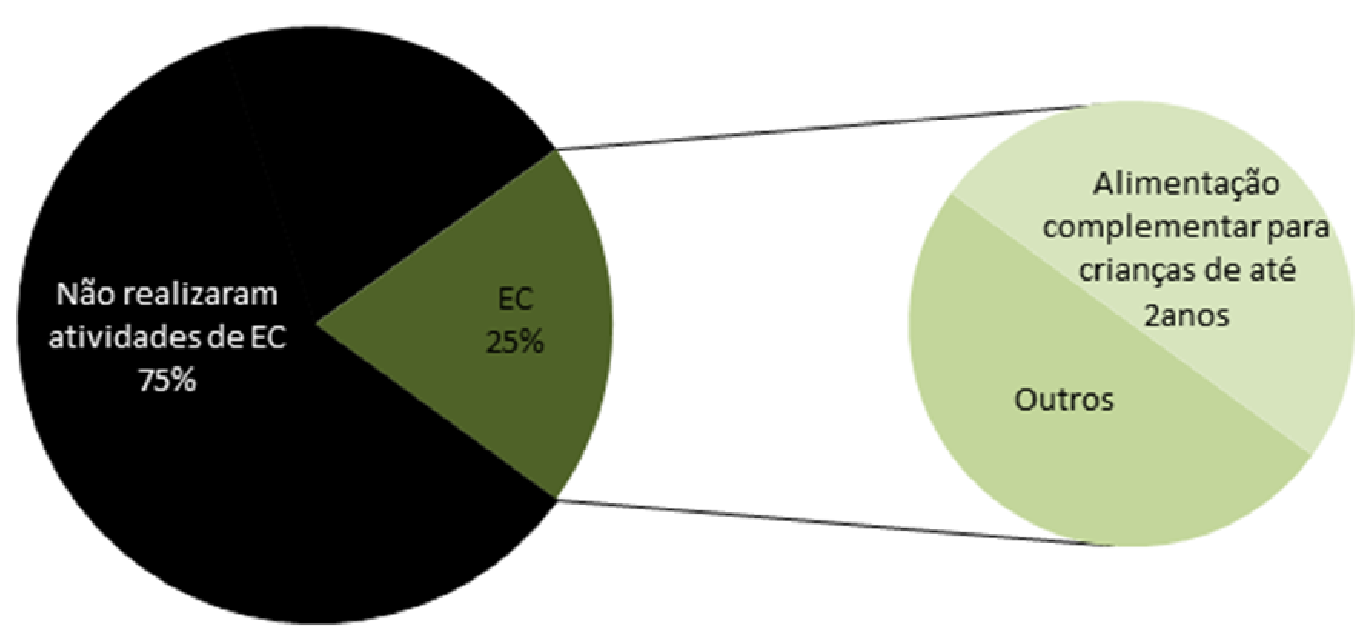

FIGURA 2 - Educação Continuada (EC) para ACS no município de Diamantina, MG, segundo a frequência de ESFs que realizaram ou não a mesma e segundo tema trabalhado, 2012.

\section{Discussão e Conclusões}

Os agentes comunitários de saúde (ACS) constituem uma importante estratégia de aprimoramento e de consolidação da ESF, pois as principais ações deste programa se dão por meio dos ACS (MINISTÉRIO DA SAÚDE, 2003). O ACS desponta como o 
elo facilitador que possibilita a confiança e

o vínculo entre a equipe de saúde e a comunidade assistida.

A análise dos artigos publicados no período de 2008 a 2012 que tratavam de educação para os Agentes Comunitários de Saúde mostrou-nos que a modalidade presencial ainda é a principal forma de capacitação destes profissionais utilizada no país. Esta escolha pode ser devida à facilidade de se incluir todos os sujeitos na atividade, uma vez que a modalidade à distância excluiria aqueles que não têm acesso à internet ou que não têm tempo disponível para se dedicar a um curso à distância.

Os cursos de capacitação e as oficinas ainda constituem preferência para a educação dos ACS. Metodologias ativas de aprendizagem não vêm sendo empregadas, embora sejam, comprovadamente, mais efetivas em relação às passivas. Uma estratégia de aprendizagem ativa poderia envolvê-los mais, aumentando seu interesse pela ação, o que lhes traria maior conhecimento. Aliado a isto, os ACS são profissionais que, na maioria das vezes, não possuem uma formação técnica que os possibilite conhecimentos básicos que lhes serão exigidos pelos usuários. Faz parte do trabalho do ACS o questionamento ao cliente sobre a adesão ao tratamento, sobre sua rotina, sobre seu estado geral de saúde. Este precisa conhecer o que é alarmante ou não, só a partir daí ele poderá dar um feedback à sua Unidade sobre a família que visitou. Segundo Nogueira (2000), o ACS ora é entendido como pertencente ao grupo de enfermagem por realizar cuidados de saúde, ora é visto como um trabalhador sui generis com identidade comunitária, que realiza tarefas que não se restringem ao campo da saúde, como por exemplo, a facilitação do acesso aos direitos de cidadania de forma geral.

Em relação aos artigos analisados, pôde-se perceber ainda uma diversidade de temas trabalhados nas ações de educação em saúde no país, sendo que São Paulo lidera como estado que mais capacitou seus ACS no quinquênio 2008-2012.

As ações de educação em saúde realizadas no município de Diamantina, semelhantemente ao que ocorre no restante do país, ainda é insuficiente, em quantidade, necessitando urgentemente de ser ampliada. Um programa de capacitação para os ACS poderia render-lhe maior competência para o desenvolvimento de seu trabalho, maior autoestima, por tornarem-se sabedores de sua capacidade, o que iria repercutir no serviço e na população. Entretanto, torna-se necessária a realização de um estudo para se definir necessidades e interesses destes profissionais. De acordo com Tomaz (2002), três aspectos devem ser levados em conta ao discutir o processo de formação ou qualificação de recursos humanos dos ACS; o perfil do profissional a ser capacitado, sua qualificação e necessidades de formação e quais as competências devem ser desenvolvidas ou adquiridas no processo educacional. $\mathrm{O}$ 
processo de qualificação dos ACS ainda é desestruturado, fragmentado, e, na maioria das vezes, insuficiente para desenvolver as novas competências necessárias para o adequado desempenho de seu papel.

Diante do exposto, a formação dos ACS não pode prescindir apenas na combinação de conteúdos que os habilitem para o reconhecimento da dinâmica social da comunidade, suas disponibilidades e necessidades, que podem ser considerados os pontos cegos, mas também com outros instrumentos para intervenções técnicas específicas. Os conhecimentos exigidos no processo de trabalho dos ACS são complexos e diversificados e transcendem o campo da saúde por requererem aprendizagem de aspectos que estão presentes nas condições de vida da população e que exigem uma atuação intersetorial (MENDONÇA, 2004).

Desta maneira, ocorre uma "superheroização" e "romantização" dos ACS quando se atribui a estes profissionais o papel de ser a "mola propulsora da consolidação do SUS". Entende-se que suas atribuições e seu papel têm sido distorcidos, sobrecarregando seu trabalho (TOMAZ, 2002).Em seu trabalho, publicado em 2002, Nunes e colaboradores referem-se à fala dos ACS que, ao compor uma equipe de saúde, consideram estar assumindo responsabilidade de dominar conteúdos e práticas referentes à biomedicina. Por meio da formação que deveriam receber, teriam acesso ao saber biomédico que, por ser mais valorizado do que o conhecimento popular, lhes conferiria maior prestígio social. Esta seria uma contradição, já que o conhecimento característico do ACS é o saber popular em saúde e o conhecimento sobre a dinâmica social da comunidade onde trabalha.

Sendo assim, Santos et al. (2011), afirmam que características como a falta de experiência, de integração com o restante da equipe e de motivação dos ACS podem comprometer a qualidade de suas ações na comunidade. É necessário que gestores e equipe de saúde repensem as práticas de saúde, para que o papel dos ACS seja realmente entendido e, sobretudo, para que possam ser mais valorizados enquanto profissionais da área da saúde. Afinal, são eles que mantêm o contato estreito com os usuários do sistema.

\section{Referências Bibliográficas}

ALVARENGA KF et al. Proposta para capacitação de agentes comunitários de saúde em saúde auditiva. Pró-Fono Revista de Atualização Científica. 2008;20(3):171-6. http://www.scielo.br/pdf/pfono/v20n3/06.pdf

ANDRADE, RD et al. Jogo educativo: capacitação de agentes comunitários de saúde sobre doenças respiratórias infantis. Acta Paul Enferm 2008;21(3):444-8 http://www.scielo.br/pdf/ape/v21n3/pt_10.pdf

BRASIL. Ministério da Saúde. Programa de Saúde da Família. Brasília; 2001. http://www.saude.gov.br/psf/programa

BRASIL. Ministério da Saúde. Secretaria de Assistência. Departamento de Assistência e Promoção da Saúde. Manual para organização da Atenção Básica. Brasília; 1999.

BRASIL. Portaria $n^{\circ} 648$ de 28 de março de 2006. Dispõe sobre a revisão de diretrizes e normas para a organização da Atenção Básica para o Programa da Saúde da Família (PSF) e o 
Programa de Agentes Comunitários de Saúde (PACS). Diário Oficial da União 2006; 28 mar.

BRASIL. Programa Saúde da Família: ampliando a cobertura para consolidar a mudança do modelo de atenção básica.Brasília: Ministério da Saúde; 2003.

BRIGAGÃO JIM, GONÇALVES R. Oficinas de promoção de saúde: discutindo os dilemas do cotidiano de um grupo de agentes comunitárias de saúde. Paideia 2009, 19 (44), 387-393 http://www.scielo.br/pdf/paideia/v19n44/a12v1 9n44.pdf

BRITES LS et al. Fonoaudiólogo e agente comunitário de saúde: uma experiência educativa. Rev Soc Bras Fonoaudiol. 2008;13(3):258-66

http://www.scielo.br/pdf/rsbf/v13n3/a10v13n3.p $\underline{\mathrm{df}}$

CARDOSO FA et al. Capacitação de agentes comunitários de saúde: experiência de ensino e prática com alunos de Enfermagem. Rev Bras Enferm, 2011; 64(5): 968-73. http://www.scielo.br/pdf/reben/v64n5/a26v64n5 .pdf

CONSELHO NACIONAL DE SAÚDE. Resolução 196/96. Diretrizes e Normas regulamentadoras de pesquisa envolvendo seres humanos. Brasília: Ministério da Saúde; 1996.

CORIOLANO MWL et al. Repercussão de uma intervenção educativa com agentes comunitários de saúde nas condições ambientais de domicílios de crianças asmáticas. J Bras Pneumol. 2011;37(3):317-325 http://www.scielo.br/pdf/jbpneu/v37n3/v37n3a0 7.pdf

DAVID HMSL, MARTELETO RM. Almanaque da dengue: leituras e narrativas de agentes Comunitários de Saúde. Rev Bras Enferm 2012;65(6):909-15. http://www.scielo.br/pdf/reben/v65n6/a04v65n6 .pdf

DIAS, MDA et al. Saúde do trabalhador na atenção básica: análise a partir deuma experiência municipal. Trab. educ. saúde [online]. 2011; 9(1): 137-148.

FELICIANO KVO et al. Avaliação continuada da educação permanente na atenção à criança na estratégia saúde da família. Rev. Bras. Saúde Matern. Infant. 2008;8(1): 45-53. http://www.scielo.br/pdf/rbsmi/v8n1/06.pdf

FRAZÃO P, MARQUES D. Efetividade de programa de agentes comunitários na promoção da saúde bucal. Rev Saúde Pública
2009;43(3):463-71.

http://www.scielo.br/pdf/rsp/v43n3/85.pdf

GARNELO L et al. Formação técnica de agente comunitário indígena de saúde : uma experiência em construção no Rio Negro. Trab Educ Saúde 2009; 7(2): 373-385. http://www.scielo.br/pdf/tes/v7n2/10.pdf

MACHADO MCHS et al. Avaliação de intervenção educativa sobre aleitamento materno dirigida a agentes comunitários de saúde Rev. Bras. Saúde Matern. Infant. 2010;10(4): 459-468. http://www.scielo.br/pdf/rbsmi/v10n4/06.pdf

MELO TM et al. Opinião dos agentes comunitários de saúde sobre o uso da videoconferência na capacitação em Saúde auditiva infantil. Rev. CEFAC 2011;13(4):692697 http://www.scielo.br/pdf/rcefac/v13n4/7010.pdf

MENDONÇA, MHM. Profissionalização e regulação da atividade do agente comunitário de saúde no contexto da reforma sanitária.Trabalho, Educação e Saúde 2004; 2(2):353-365.

NASCIMENTO EPL, CORREA CRS. O agente comunitário de saúde: formação, inserção e práticas. Cad. Saúde Pública 2008;24(6):13041313

http://www.scielosp.org/pdf/csp/v24n6/11.pdf

NOGUEIRA, RP, SILVA F, Ramos Z. A vinculação institucional de um trabalhador sui generis o agente comunitário de saúde. [Textos para discussão $\mathrm{n}^{\mathrm{o}}$ 735]. 2000. Disponível em: http:///www.ipea.gov.br

NUNES, MO et al. O agente comunitário de saúde: construção da identidade desse personagem híbrido e polifônico.Cad Saúde Pública 2002; 18(6):1639-1646.

PAIXÃO PBS et al. A prática de alfabetização em Informação e Comunicação em Saúde: o olhar dos agentes comunitários de Saúde sobre o projeto de Inclusão Digital em Sergipe, Bras. Interface - Comunic Saúde Educ 2008;15(38):937-946.

http://www.scielo.br/pdf/icse/v15n38/29.pdf

SANTOS, KT et al. Agente comunitário de saúde: perfil adequado a realidade do Programa Saúde da Família?. Ciênc. saúde coletiva [online]. 2011, 16(1), 1023-1028. http://dx.doi.org/10.1590/S141381232011000700035.

SERPA-FILHO A, OKOCHI RCN. Capacitação de técnicos de saúde: uma experiência pioneira no estado do Tocantins, Brasil. Trab Educ 
Saúde $\quad 2010 ; \quad 8(2): \quad$ 319-355. http://www.scielo.br/pdf/tes/v8n2/a09v8n2.pdf

SILVA CA et al. Formação técnica do agente comunitário de saúde: desafios e conquista da Escola Técnica de Saúde do Tocantins. Trab Educ Saúde 2010; 7(3): 609-621. http://www.scielo.br/pdf/tes/v7n3/14.pdf

SILVA CB et al. Estratégia de Apoio em Saúde Mental aos Agentes Comunitários de Saúde de Salvador-BA. Saúde Soc. 2012; 21(1):153-160. http://www.scielo.br/pdf/sausoc/v21n1/15.pdf

SILVA TL et al. Capacitação do Agente Comunitário de Saúde na Prevenção do Câncer de Colo Uterino. Rev Bras Educ Médica 2012;36 (1) $155-160$ http://www.scielo.br/pdf/rbem/v36n1s1/v36n1s 1a21.pdf

SILVA, JA, DALMASO, ASW. O agente comunitário de saúde e suas atribuições: os desafios para os processos de formação de recursos humanos em saúde. Interface Comunic, Saúde, Educ 2002; 6(10):75-83.

SOUSA MF, PARREIRA CMSF. Ambientes verdes e saudáveis: formação dos agentes comunitários de saúde na Cidade de São Paulo, Brasil. Rev Panam Salud Publica. 2010;28(5):399-404.

http://www.scielosp.org/pdf/rpsp/v28n5/10.pdf

TOMAZ, JBC. O agente comunitário de saúde não deve ser um "super-herói".Interface Comunic, Saúde, Educ 2002; 6 (10):84-87. 